SAMMLUNG TUSCULUM

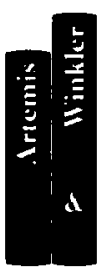


Wissenschaftliche Beratung:

Karl Bayer, Manfred Fuhrmann, Fritz Graf.

Erik Hornung, Rainer Nickel 
PROPERZ TIBULL

\section{LIEBESELEGIEN CARMINA}

Lateinisch-Deutsch

Neu herausgegeben und übersetzt von Georg Luck 
Die Liebeselegien von Properz und Tibull sind erstmals 1964 von Georg Luck in der * Bibliothek der Alten Welt= herausgegeben worden. Die vorliegende Ausgabe stellt eine komplette Neubearbeitung dar.

Die Deutsche Bibliothek - CIP-Einheitsaufnahme

Propertius, Sextus: [Elegiae]

Liebeselegien / Properz. Carmina / Tibull. lateinisch-deutsch.

Hrsg. und übers. von Georg Luck.

1. Aufl. - Zürich : Artemis und Winkler, 1996

(Sammlung Tusculum) ISBN 3-7608-1689-4

NE: Luck, Georg [Hrsg.]; Tibullus, Albius: Carmina

Artemis \& Winkler Verlag Zürich / Düsseldorf

○ 1996 Artemis Verlags AG Zürich

Alle Rechte, einschließlich derjenigen

des auszugsweisen Abdrucks, der fotomechanischen und elektronischen Wiedergabe, vorbehalten

Satz: Jung Satzcentrum, Lahnau Druck und Bindung: Pustet, Regensburg Printed in Germany 